\title{
Bilateral Striopallidodentate Calcinosis in Female: A case report
}

\author{
Arun Kadel ${ }^{1}$, Avinash Chandra ${ }^{2}$,Reema Rajbhandari ${ }^{2}$, Pranaya Shrestha ${ }^{3}$, Pravesh Rajbhandari ${ }^{3}$ \\ ${ }^{1}$ Department of Internal Medicine, Kathmandu Model Hospital, Kathmandu, Nepal \\ ${ }^{2}$ Department of Neurology, Annapurna Neurological Institute and Allied Sciences, Maitighar, Kathmandu, Nepal \\ ${ }^{3}$ Department of Neurosurgery, Annapurna Neurological Institute and Allied Sciences, Maitighar, Kathmandu, Nepal
}

\section{CORRESPONDENCE}

\author{
Dr. Arun Kadel \\ Department of Internal \\ Medicine, Kathmandu Model \\ Hospital,Kathmandu,Nepal \\ Email:arun.kadel@yahoo.com
}

\section{ARTICLE INFO}

Article History

Submitted: 29 November, 2020

Accepted: 25 December, 2020

Published: 8 February,2021

Source of support: None

Conflict of Interest: None

Copyright : (CThe Author(S) 2020

This is an open access article under the Creative Common Attribution license CC-BY 4.0

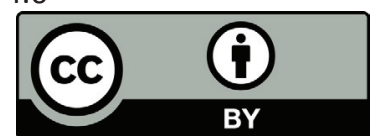

\begin{abstract}
Bilateral striopallidodentate calcinosis (BSPDC) is associated with many neurological and psychiatric abnormalities and most commonly present with extra pyramidal symptoms and can be idiopathic or associated with endocrinopathy, frequently with parathyroid disorders. Here we describe a case who presented with generalized seizure. During workup, the cause of seizure was found to be bilateral and symmetric, extensive, irregular, intraparenchymal calcifications involving the basal ganglia, thalamus and dentate nucleus, white matter in the frontal, parietal, and occipital lobes and dentate nuclei of cerebellum. On the basis of clinical features, investigations, and exclusion of other causes of intracranial calcification a clinical diagnosis of BSPDC was made. BSPDC should be considered in the differential diagnosis of endocrinopathy particularly parathyroid disorders, when associated with neurological and psychiatric abnormalities.
\end{abstract}

Key words: Bilateral striopallidodentate calcinosis; Fahr's disease Cerebral calcification; Basal ganglia; Hypoparathyroidism.

\section{INTRODUCTION}

Blateral striopallidodentate calcinosis (BSPDC), popularly referred to as Fahr's disease, is a condition characterized by the occurrence of vascular non-arteriosclerotic bilateral calcification in the cortex, in the basal ganglia and in the dentate nucleus of the cerebellum. It is an autosomal dominant inheritance to pseudo-pseudohy poparathy roidism, degenerative disordercharacterized clinically from asymptomatic to multiple neurological and psychiatric symptoms occurring secondary to calcification in brain parenchyma with subsequent neuronal loss. ${ }^{1}$ It may begin early in life with progressive dementia, convulsions and rigidity. In CT of 2318 patients with Fahr's disease 12.5\% have calcifications in basal ganglia. The most frequently seen localization is globus pallidus with a rate of $96.4 \% .2$

\section{CASE REPORT}

A 30 years female presented with 2 months history of severe headache, intermittent type, few episodes per day associated with stiffness of body, tongue bite, but no abnormal movements, frothing, unrolling of her eyes. She also had complaints of dizziness. Her past medical history was insignificant. Clinical examination revealed normal orientation to time, place and person with normal level of consciousness. Physical examination was unremarkable. Laboratory examinations including blood levels of glucose, Renal function test, Liver function test, thyroid function test were within normal limits. Parathyroid hormone, serum calcitonin and serum phosphorus were high whereas serum vitamin $D$, serum calcium, urinary calcium were low. ECG and EEG examinations were unremarkable. Abdominal ultrasound was normal. NCCT brain revealed bilateral and symmetric, extensive, irregular, intraparenchymal calcifications involving the basal ganglia, thalamus and dentate nucleus, white matter in the frontal, parietal, and occipital lobes and dentate nuclei of cerebellum. The CT scan findings when correlated with clinical history and blood chemistry a diagnosis of bilateral striopallidodentate calcinosis (BSPDC) was made. 
with clinical history and blood chemistry a diagnosis of bilateral striopallidodentate calcinosis (BSPDC) was made. Endocrinologist consultation was done for biochemistry derangement; Pseudohypoparathyroidism was diagnosed and advised for vitamin D and calcium supplementation. Anti-convulsant was also started and during follow up after 3 months patients was seizure free without any fresh complaints.

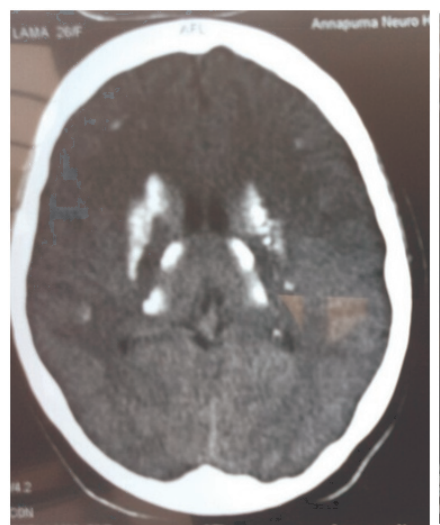

Figure 1

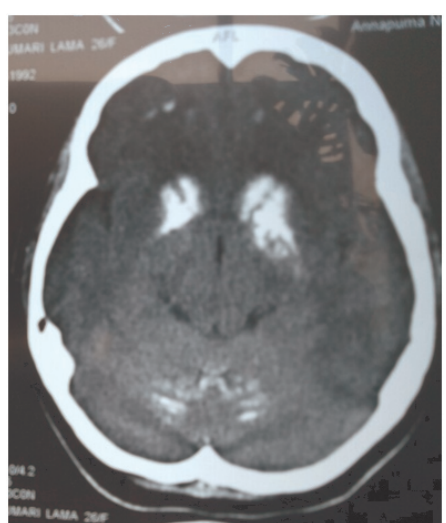

Figure 2
Figure 1 and Figure 2: Plain computed Tomography brain scan reveals extensive bilateral calcification of basal ganglia, thalamus white matter in the frontal, parietal, and occipital lobes and dentate nuclei of cerebellum.

\section{DISCUSSION}

Fahr's syndrome corresponds to mineral deposits in the blood vessel wall in the cortex, in the basal ganglia and in the dentate nucleus of the cerebellum. Though there are many etiologies found but are dominated mainly by hypoparathyroidism. In the presence of neuropsychiatric manifestations associated with calcifications of the basal ganglia, it is imperative to look for phosphocalcic metabolic disorders, in order to detect hypoparathyroidism and thus adopt the most appropriate therapeutic measures. The clinical features of 99 patients suffering from BSPDC have been summarized in a registry. ${ }^{3} 67$ were symptomatic and 32 were asymptomatic. Movement disorders accounted for $55 \%$ of the total symptomatic patients.Of the movement disorders, Parkinsonism accounted for the most followed by chorea, tremor, dystonia, athetosis, and orofacial dyskinesia and other neurologic manifestations included: cognitive impairment, cerebellar signs, speech disorder, pyramidal signs, psychiatric features, gait disorders, sensory changes, and pain. Patients who become symptomatic early in adulthood are more likely to present with psychosis, whereas those presenting later in life typically manifest dementia and a motor system disorder. There is no cure for Fahr's disease but the treatment is towards minimizing the symptoms. Most cases present with extra pyramidal symptoms. ${ }^{4}$ Here we described a case of Fahr's disease, who presented with complex partial seizure and behavioral abnormalities and the cause of seizure was found to be bilateral calcification of basal ganglia and thalamus, cerebellum due to abnormal calcium and phosphate metabolism. We made a clinical diagnosis of complex-partial seizure secondary to Fahr's disease on the basis of clinical features, investigations, and exclusion of other causes of intracranial calcification. We started anti-convulsants on our patient and advised for follow-up. Patient became symptoms free thereafter and no progression of calcification was found. Hoque MA and colleagues reported a similar case of seizure disorder secondary to Fahr's disease. ${ }^{5}$

\section{CONCLUSION}

BSPDC is a neurodegenerative syndrome that is associated with symmetric, intracerebral calcifications mainly in the basal ganglia. It is not clear whether the calcification is a metastatic deposition, secondary to local disruption of blood brain barrier, or is due to disorder of neuronal calcium metabolism. BSPDC should be considered in the differential diagnosis of endocrinopathy particularly parathyroid disorders, when associated with neurological and psychiatric abnormalities. Age of onset is typically in the 30 s to 50 s, although it can occur at any time in childhood or adolescence. There is no cure nor is there standard course of treatment for BSPDC and treatment addresses symptoms on an individual basis. The prognosis is variable and unpredictable.

\section{REFERENCES}

1. Manyam BV. What is and what is not 'Fahr's disease'. Parkinsonism \& related disorders. 2005 Mar 1;11(2):7380.

2. Gomille T, Meyer RA, Falkai P, Gaebel W, Königshausen T, Christ F. Prevalence and clinical significance of computerized tomography verified idiopathic calcinosis of the basal ganglia. Der Radiologe. 2001 Feb;41(2):205-10.

3. Manyam BV, Walters AS, Narla KR. Bilateral striopallidodentate calcinosis: clinical characteristics of patients seen in a registry. Movement disorders: official journal of the Movement Disorder Society. 2001 Mar;16(2):258-64

4. Ahad MA, Bala CS, Karim SR. Fahr's syndrome. Bangladesh Medical Journal Khulna. 2012;45(1-2):33-5.

5. Hoque MA, Siddiqui MR, Arafat Y, Khan SU, Rahman KM, Mondol BA, Mohammad QD. Fahr's disease: a very rare cause of epilepsy. Mymensingh medical journal: MMJ. 2010 Jan;19(1):127-9. 